\title{
EMC-Aware Analysis and Design of a Low-Cost Receiver Circuit under Injection Locking and Pulling
}

\author{
Martijn Huynen, Gert-Jan Stockman, Frederick Declercq, Guy Torfs, \\ Johan Bauwelinck and Dries Vande Ginste \\ Department of Information Technology, Ghent University/iMinds, Sint-Pietersnieuwstraat 41, B-9000, Gent, Belgium.
}

\begin{abstract}
In low-cost receiver applications, the preselect filter is often omitted in order to reduce the footprint of the total system. However, the immunity of the receiver can be severely compromised by this approach. This paper focuses on the effects of co-located sources on the local oscillator (LO), specifically injection locking and pulling. To this end, a low-cost radio receiver $(\mathrm{RF})$ front-end is designed for operation in the $2.45 \mathrm{GHz}$ industrial, scientific and medical (ISM) radio band. In addition to the effects on the oscillator, the consequences on the receiver's performance are evaluated as well. For the first time in literature, this work demonstrates the critical necessity to take the potentially detrimental effects caused by injection locking and pulling into account during Electromagnetic Compatibility (EMC)-aware design.
\end{abstract}

\section{INTRODUCTION}

Over the last few decades, an explosive growth in wireless communication has been witnessed. Together with the rise of smarthphones and other multimedia applications, the demand for low-cost communication circuits has increased drastically. In an attempt to reduce the cost of a standard superheterodyne receiver [1], the radio frequency $(\mathrm{RF})$ preselect filter is often omitted [2]. This measure should not be taken lightly, however, as it can affect the immunity of the receiver system to interfering signals, as already discussed in [3]. Moreover, as shown in the present contribution, when out-of-band signals (for the intended application), having a frequency in the vicinity of the local oscillator (LO) frequency, leak into the oscillator, they can cause injection locking and/or pulling. These nonlinear effects have the potential to jeopardize the proper operation of the total receiving chain [4].

In this contribution, the goal is to analyze and design a low-cost radio frequency (RF) receiver front-end, intended for use in the $2.45 \mathrm{GHz}$ industrial, scientific and medical (ISM) radio band (see Fig. 1). Subsequently, the system is subjected to co-located noise sources $\left(f_{c o}\right)$ to induce injection locking and pulling and to evaluate the receiver's performance under these conditions. It is shown that EMC-aware design needs to encompass rigorous theoretical analysis, thorough simulation, and careful prototyping and measurements.

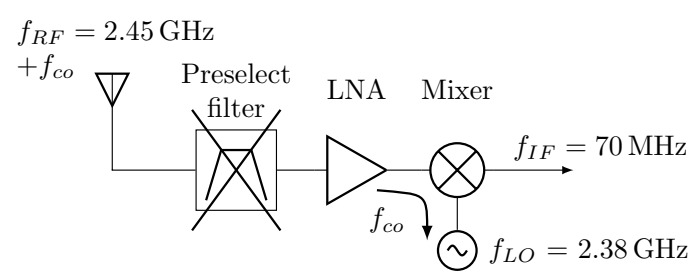

Fig. 1. Low-cost radio frequency (RF) front-end. The desired signal at frequency $f_{R F}$ and the interfering noise at frequency $f_{c o}$ are both unfiltered, amplified by means of a low noise amplifier (LNA), and downconverted to the intermediate frequency (IF).

This paper starts off with a short introduction to injection locking and pulling in order to get a solid grasp of their major properties (Section II). The design of the different building blocks is discussed in Section III. Measurements on the oscillator and the total system are described and interpreted in Section IV. Conclusions are outlined in Section V.

\section{InJECTION LOCKING AND PULling}

Injecting a signal with a frequency close to the oscillator's natural frequency can cause injection locking or pulling. When the injected frequency is within a certain frequency range around the natural frequency, the so-called lock range, the oscillator's frequency will change to the frequency of the external signal. This frequency shifting phenomenon is called injection locking. The width of the lock range is proportional to the amplitude of the injected signal, the natural frequency of the oscillator and inversely proportional to the quality factor (Q) of the oscillator, a measure for the frequency selectivity [5].

Bringing the external frequency outside of the lock range does not result in an immediate return to the natural behavior of the oscillator. Quite the opposite, the external signal will still influence the oscillator's frequency response by pulling the frequency towards its own. As a result, the oscillator's frequency will vary periodically by going through several stages. It will attempt to lock to the external signal at first but as this situation cannot be maintained, the frequency will rapidly fall back towards the natural frequency. However, the instantaneous frequency overshoots this point and promptly returns towards the external frequency to attempt locking on 


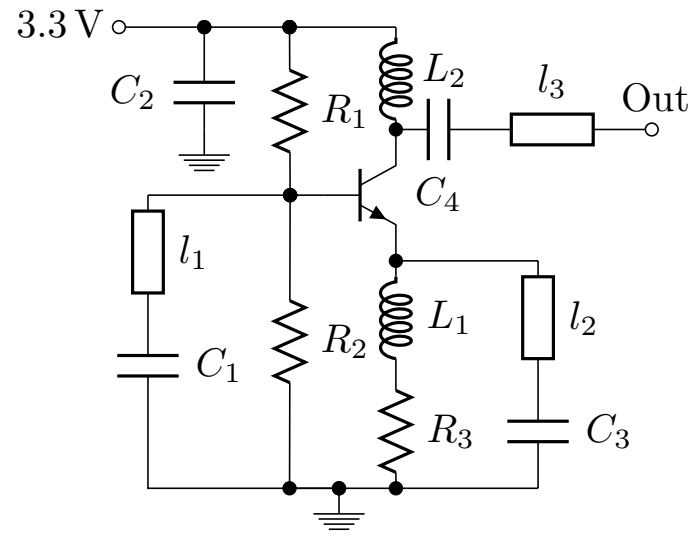

Fig. 2. Schematic of the oscillator design. The transmission lines are microstrip lines with length $l_{i}$ and width $w_{i}(i=1,2,3)$.

once again. To put it briefly, the oscillator's frequency has become time-dependent due to the pulling effect of the external signal, hence the name injection pulling. This response manifests itself in the frequency domain by the appearance of a lot of progressively diminishing, tightly spaced frequency components close to the external frequency which are, moreover, mainly present at one side of the external frequency. The further one departs from the lock range, the less pronounced the effect becomes whereby the natural frequency will start prevailing once more.

\section{DESIGN OF A RECEIVER CIRCUIT}

\section{A. Negative resistance oscillator}

One possible view on oscillators that is convenient for high operating frequencies is that of the negative resistance oscillator. A potentially unstable amplifier can be represented by a one-port network that delivers a complex input impedance with a negative real part at its port. This negative impedance [6] is crucial to obtain an oscillator as it denotes the ability of the circuit to overcome the losses of the components and as such to ensure a stable oscillation. The frequency can subsequently be fixed by compensating the input impedance (including the imaginary part) with a matching network to the load. A design of such an oscillator was made on a DE104 substrate by Elprinta (thickness of $800 \mu \mathrm{m}, \epsilon_{r}=4.75$ and $\tan \delta=0.02$ ) with a bipolar junction transistor (BJT), i.e. the BFP183 by Infineon Technologies as the active element. Furthermore, transmission lines were employed to ensure a proper frequency selectivity of the oscillator. Table I together with Fig. 2 depicts the oscillator that produces a ground tone at $2.344 \mathrm{GHz}$ with a power of $9 \mathrm{dBm}$ for a supply voltage of $3.3 \mathrm{~V}$ and a load of $50 \Omega$. The first two overtones are substantially suppressed by $13.5 \mathrm{~dB}$ and $17.5 \mathrm{~dB}$, respectively.

\section{B. Diode mixer}

A passive mixer consisting of a Mini Circuits ADE-35+ double balanced diode mixer with a BP2U+ power splitter
TABLE I

COMPONENT VALUES AND TRANSMISSION LINE DIMENSIONS OF THE OSCILLATOR.

\begin{tabular}{c|c||c|c} 
Component & Value & Component & Value \\
\hline$R_{1}$ & $1500 \Omega$ & $l_{1} / w_{1}$ & $35 \mathrm{~mm} / 0.9 \mathrm{~mm}$ \\
$R_{2}$ & $820 \Omega$ & $l_{2} / w_{2}$ & $19.6 \mathrm{~mm} / 0.9 \mathrm{~mm}$ \\
$R_{3}$ & $27 \Omega$ & $l_{3} / w_{3}$ & $10.2 \mathrm{~mm} / 1.416 \mathrm{~mm}$ \\
\hline$L_{1}$ & $37 \mathrm{nH}$ & $L_{2}$ & $37 \mathrm{nH}$ \\
\hline$C_{1}$ & $52.1 \mathrm{pF}$ & $C_{3}$ & $52.1 \mathrm{pF}$ \\
$C_{2}$ & $100 \mathrm{pF}$ & $C_{4}$ & $27 \mathrm{pF}$
\end{tabular}

(with one terminated port) at its LO input was used to perform the downconversion from the RF signal to an appropriate intermediate frequency (IF). The circuit exhibits a conversion loss of $7.5 \mathrm{~dB}$ and an RF to LO isolation of about $25 \mathrm{~dB}$. This isolation lowers the effective power of undesired co-located sources that reaches the oscillator.

\section{MeAsurements}

Measurements are performed using an N5242A PNA-X from Agilent Technologies to generate one single tone or a two-tone signal, depending on the exact measurement. Frequency spectra are captured using both a Rhode \& Schwarz FSV40 signal analyzer and the aforementioned network analyzer. In order to measure the LO spectrum while it was embedded in the system, a quadrature hybrid, i.e. a directional coupler, was used [7].

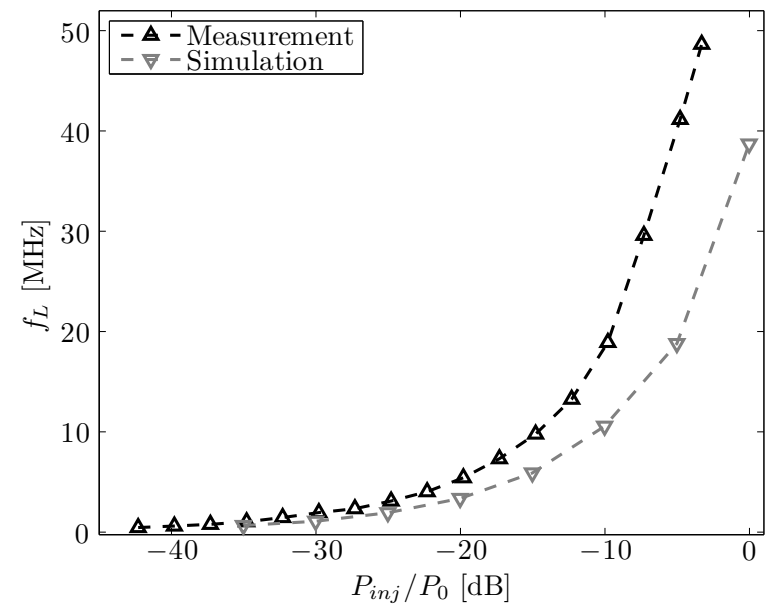

Fig. 3. Lock range of the stand-alone oscillator.

\section{A. Oscillator}

The first experiments were performed on the oscillator itself to characterize the effects of injection locking and pulling without the influence of the mixer. As the lock range is the most notable characteristic of a system under injection locking/pulling, the one-sided lock range $\left(f_{L}\right)$ was measured first as a function of the ratio of the injected power $\left(P_{i n j}\right)$ to the oscillator's output power $\left(P_{0}\right)$ with the injection point 


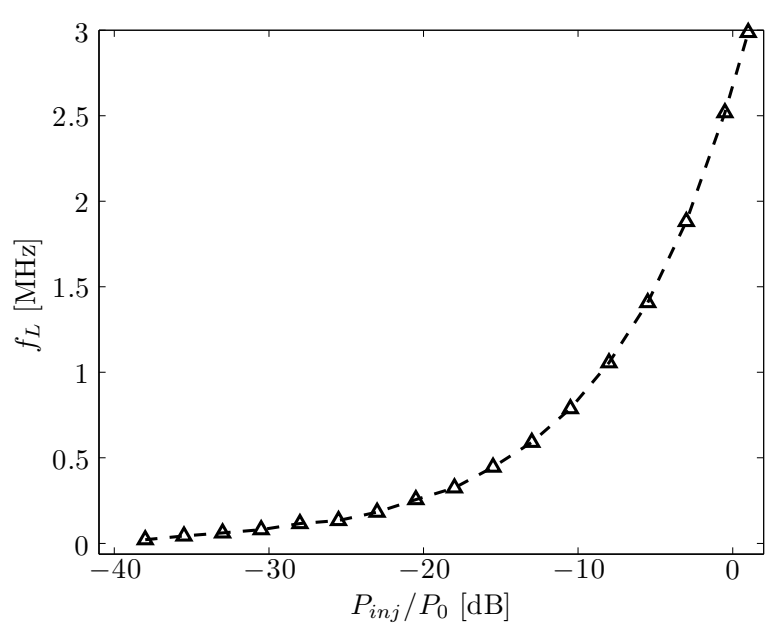

Fig. 4. Lock range with injection via the power supply.

being the output of the oscillator. Fig. 3 depicts the lock range for both measurements and simulation. For small injected powers, the lock range is clearly very small but when the power starts to increase, it grows drastically. Simulation results tend to predict a smaller lock range than was actually observed which is caused by its higher $\mathrm{Q}$ factor as not all parasitic effects can be included in a simulation.

A disturbance could potentially enter the system in a second way, i.e. via the power supply. This situation was tested using a Picosecond Pulse Labs bias tee to combine the power supply voltage and the disturbance signal. Using a similar approach as employed above, the lock range was measured as depicted in Fig. 4. Although the lock range exhibits the same qualitative behavior as before, it is obvious that the circuit is much more robust for this injection point as the lock range is about twenty times smaller. This robustness is caused by decoupling capacitor $C_{2}$.

Although the lock range is a first indication for the range of potentially critical frequencies, one must not forget that the second phenomenon, i.e. injection pulling, falls outside this lock range. The effects of injection pulling are most notable at the edges of the lock range and fade away as the frequency of the injected signal $\left(f_{i n j}\right)$ moves away from the natural frequency. Fig. 5 shows the spectrum of the oscillator's output for $P_{i n j}=-20 \mathrm{dBm}$ and injection via the power supply. The frequency of the disturbance lies 8.42 times half the lock range above the natural frequency, i.e. well above the upper bound of this lock range. Despite this distance from the lock range, the oscillator clearly suffers from substantial injection pulling effects. The dominant component has shifted back towards the natural frequency, i.e. $2.34645 \mathrm{GHz}$, but still differs by a small fraction and some considerable components are observed below the natural frequency. One can thus see that although the lock range itself may be quite small as compared to the natural frequency, the area in which the oscillator's spectrum is severely degraded by injection pulling, is approximately one order of magnitude larger. Moreover, it is clear that even for

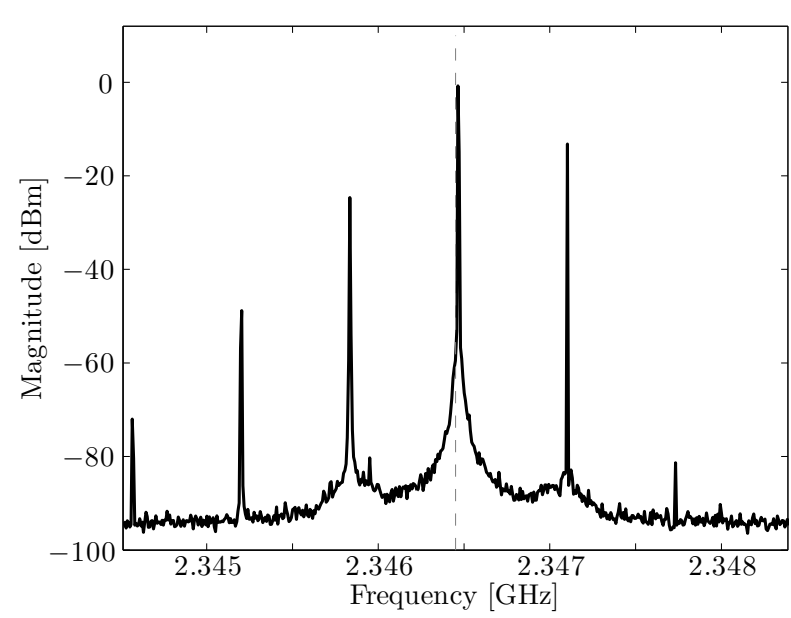

Fig. 5. Output spectrum for $P_{i n j}=-20 \mathrm{dBm}$ and $f_{i n j}=f_{0}+8.42 \cdot f_{L}$.

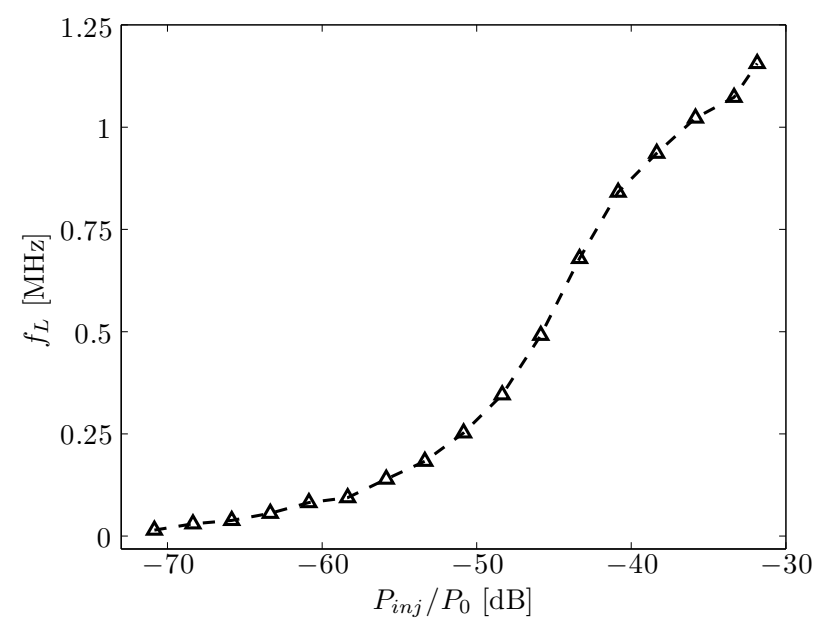

Fig. 6. Lock range of the total receiver front-end.

weak noise signals, this pulling may already occur.

\section{B. Receiver front-end}

The low-noise amplifier (LNA) is assumed to have a sufficient bandwidth so as not to substantially affect the interfering signal and its influence on the oscillator. Therefore, measurements are performed on the most fundamental receiver frontend, viz. a mixer and an LO. In order to evaluate the influence of the mixer on the system's performance under injection locking and pulling, the lock range was measured once again and is depicted in Fig. 6. One notices the small injected powers as the mixer isolation and the directional coupler's losses seriously lower the effective power that reaches the oscillator. This results in a smaller lock range as compared to Fig. 3. For higher injected powers, one also notices a deviation from the anticipated behavior which is caused by changing load conditions; as the mixer has to handle larger powers, the impedances presented at its ports start to change. This variation 


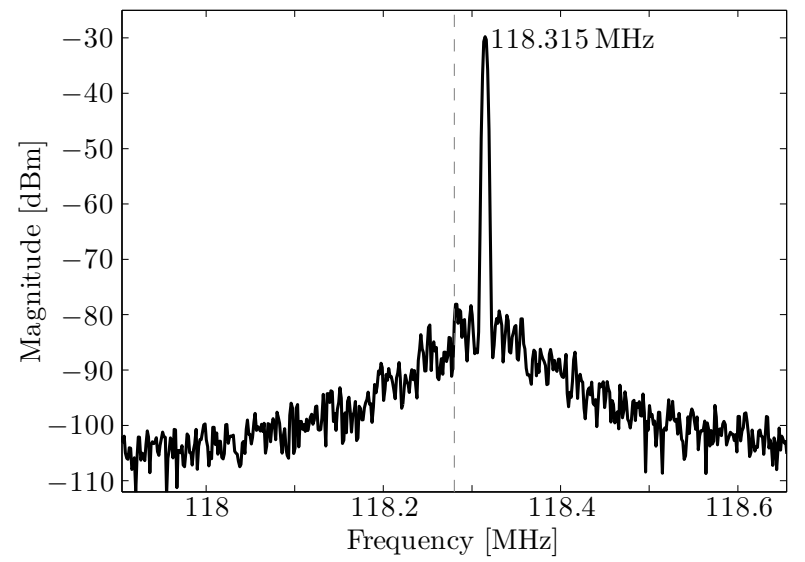

Fig. 7. IF spectrum for $f_{c o}=2.331685 \mathrm{GHz}$, i.e. the edge of the lock range.

affects the operation of the oscillator and its susceptibility to the interfering signal.

Next, the system's performance was evaluated when illuminated by multiple frequencies. A two-tone signal was inserted into the mixer via the RF port. The first tone represents the useful information signal $\left(f_{R F}\right)$ at $2.45 \mathrm{GHz}$ while the second tone plays the role of an interfering noise signal at a variable frequency $\left(f_{c o}\right)$, this to approximate a realistic scenario. Both had an input power of $-20 \mathrm{dBm}$. Figs. 7 and 8 show the IF spectrum for different values of $f_{c o}$.

As the exact LO frequency in the total system is $2.3317 \mathrm{GHz}$, the nominal, desired IF frequency for the information signal should be $118.3 \mathrm{MHz}$, indicated in both figures by the dashed vertical line. At the edge of the lock range (Fig. 7), the spectrum consists of a single frequency component which has shifted to another IF frequency. This frequency exactly corresponds to $f_{R F}-f_{c o}$, indicating that the LO is locked to $f_{c o}$. Depending on the building blocks after the front-end, this shift can cause problems if not dealt with properly.

The spectrum in the injection pulling region (Fig. 8) differs completely from the one in the injection locking situation [8]; the spectrum consists of a lot of closely spaced components, placed asymmetrically around the dominant component, which is in turn pulled away from the natural frequency. Extracting the useful information from this IF spectrum is clearly impossible as the tightly spaced frequency components inhibit proper spectral filtering. To put it briefly, the time-dependent oscillator has ruined the proper operation of the RF receiver front-end.

For stronger interference signals, the lock range and thus the frequency range in which the operation is disturbed, increases. The higher power levels degrade the linearity of the mixer as well, giving rise to additional and stronger intermodulation products that further obstruct the receiver's operation. These extra frequency components clutter the IF spectrum even more and thus hinder the operation of the receiver in a much larger range.

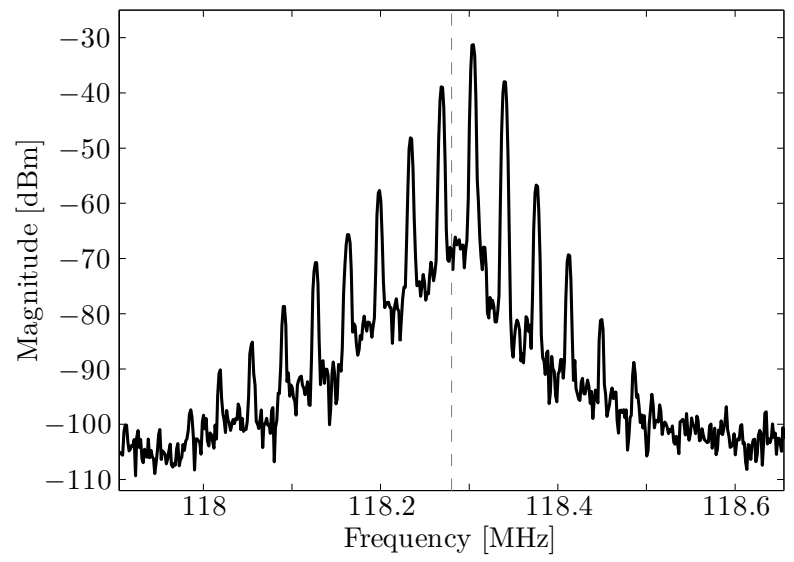

Fig. 8. IF spectrum for $f_{c o}=2.33166 \mathrm{GHz}$, causing strong injection pulling.

\section{CONCLUSIONS}

The design and EMC analysis of a low-cost RF receiver front-end, intended for use in the $2.45 \mathrm{GHz}$ ISM band, is presented in this work. The operation of both the local oscillator and the total front-end under the illumination of an interfering signal that induced injection locking and pulling was characterized. The lock range exhibits a strong influence on the injected power level as well as a high sensitivity to the point of the injection. Furthermore, it is shown that although the lock range is quite small, only a fraction of the natural frequency in a real system, the pulling effects can be detrimental to the proper functioning of the receiver frontend, even for small injected powers. As such, it is clearly shown that EMC-aware design critically depends on a design strategy that can take these nonlinear effects into account. As with most nonlinear systems, general design guidelines are hard to define. Clearly, a (large, expensive) mixer with a high $\mathrm{RF}$ to $\mathrm{LO}$ isolation may improve the immunity of the receiver front-end. Nonetheless, in some cases and depending on the (expected) presence of co-located noise sources, the preselect filter cannot simply be omitted.

\section{REFERENCES}

[1] J. Laskar, B. Matinpour, and S. Chakraborty, Modern Receiver FrontEnds: Systems, Circuits and Integration. John Wiley \& Sons, 2004.

[2] A. Rembovsky, A. Ashikhmin, V. Kozmin, and S. M. Smolskiy, Radio Monitoring: Problems, Methods and Equipment, 1st ed. Springer, 2009.

[3] A. Biondi, H. Rogier, D. Vande Ginste, and D. De Zutter, "Multi-tone EMC testing strategy for RF-devices," in IEEE 2012 Electrical Design of Advanced Packaging and Systems Symposium (EDAPS), Taipei, Taiwan, Dec. 2012, pp. 89-92.

[4] R. Adler, "A Study of Locking Phenomena in Oscillators," Proc. IRE, vol. 34, pp. 351-357, Jun. 1946.

[5] B. Razavi, "A study of Injection Locking and Pulling in Oscillators," IEEE J. Solid-State Circuits, vol. 39, no. 9, pp. 1415-1424, Sep. 2004.

[6] F. Ellinger, Radio Frequency Integrated Circuits and Technologies. Springer Science \& Business Media, 2008.

[7] D. M. Pozar, Microwave Engineering, 4th ed. New York: John Wiley \& Sons, 2012.

[8] I. Ali, A. Banerjee, A. Mukherjee, and B. N. Biswas, "Study of Injection Locking With Amplitude Perturbation and Its Effect on Pulling of Oscillator," IEEE Trans. Circuits Syst. I, vol. 59, no. 1, pp. 137-147, Jan. 2012. 